\title{
Hiding Information in Multiple Level-line Moirés
}

\author{
Thomas Walger \\ Roger David Hersch \\ École Polytechnique Fédérale de Lausanne (EPFL), Switzerland \\ \{thomas.walger, rd.hersch\}@epfl.ch
}

\begin{abstract}
Secure documents often comprise an information layer that is hard to reproduce. Moiré techniques for the prevention of counterfeiting rely on the superposition of an array of transparent lines or microlenses on top of a base layer containing hidden information. Level-line moirés consist of shapes that appear to be beating upon relative translation of a revealing grating on top of a base, in which the desired information is encoded. Usually, the base only contains the information corresponding to one moiré. In order to increase the difficulty of counterfeiting, we use tessellations to incorporate two or more moirés within the same layer. With the method we propose, the information corresponding to up to seven level-line moirés can be embedded within a single base layer. The moirés are recovered with a revealer printed on a transparency or with an array of cylindrical lenses. This method is general and can be extended to other fabrication technologies.
\end{abstract}

\section{Keywords}

Security printing; moiré; tessellation; tiling; level-line moiré

\section{INTRODUCTION}

The superimposition of gratings often yields astonishing visual effects. Some of these phenomena can easily be observed in the daily life. Moiré fringes can appear when two railings are seen one behind the other, on some translucent curtains or when you photograph a digital screen. In general, moiré effects are undesired when scanning, printing or photographing. However, moiré can also be deliberately used by artists [6] or scientists [1], [4], [5], [7] in order to create surprising effects. In the field of document security, moirés are advantageous since macroscopic effects are produced by superposing layers of microscopic structures. When generated at very high resolutions, these microstructures cannot be easily reproduced. Level-line moirés rely on a theorem from the moiré theory that states that when locally shifting a grating of lines in proportion to an elevation profile and

Permission to make digital or hard copies of all or part of this work for personal or classroom use is granted without fee provided that copies are not made or distributed for profit or commercial advantage and that copies bear this notice and the full citation on the first page. Copyrights for components of this work owned by others than ACM must be honored. Abstracting with credit is permitted. To copy otherwise, or republish, to post on servers or to redistribute to lists, requires prior specific permission and/or a fee. Request permissions from Permissions@acm.org.

DocEng'15, September 8-11, 2015, Lausanne, Switzerland.

(C) 2015 ACM. ISBN 978-1-4503-3307-8/15/09 ...\$15.00.

DOI: http://dx.doi.org/10.1145/2682571.2797078.

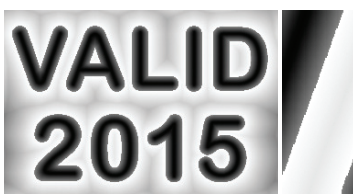

a

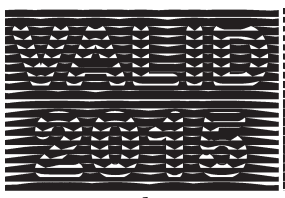

$\mathrm{d}$

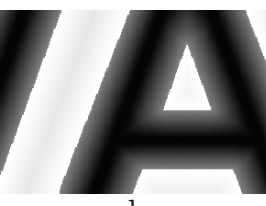

$\mathrm{b}$

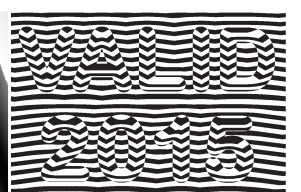

$\mathrm{C}$

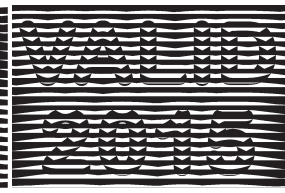

e

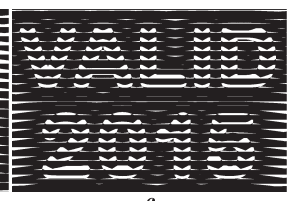

f
Figure 1: (a) Elevation profile. (b) Enlarged portion of the elevation profile. (c) Base grating. (d), (e) and (f) Levelline moiré obtained for three different translations of the revealer (resp. 0, 20 and 35 pixels upwards). The revealer has a period of 40 pixels and an aperture of 20 pixels.

superposing it with the unshifted grating, the moiré that appears is formed by the level lines of the input elevation profile [2]. Level-line moirés result in shapes that appear to be beating upon displacement of the revealer (see Figure 1). Beginning with two similar gratings, we apply local shifts to one of them (the base) according to the intensity levels of a greyscale image, which we call the elevation profile. The moiré obtained when superposing the two gratings shows level lines of the elevation profile. These level lines follow the gradient of the elevation profile when we translate the revealer. In the context of document security, our goal is to embed several layers of level-line moiré information within a single base. By tessellating the plane with hexagons and thus creating a mask for each layer of information, we can incorporate multiple level-line moirés within one base (see Figure 8). Each moiré appears when a specific revealer is superposed onto the base. The corresponding level lines move when displacing the revealer in its direction of periodicity. We try to maximise the visibility of the level-line moirés while embedding as many moiré information layers as possible. We will therefore express the trade-offs associated with the size of the tiling hexagons, the grating period and the size of the moiré-shape features.

\section{MULTIPLE LEVEL-LINE MOIRÉS}

To obtain a moiré, a base and a revealer have to be superposed. If several moirés must be obtained, we still have to superpose base and revealer but this time, we must prevent 
the revealer from exposing the information contained in the other moirés.

For this purpose, as proposed in [2], we superpose bases that have been designed to be revealed by revealers that do not interfere. In this way, each revealer reveals its own moiré. As an example, if we want to embed three level-line moirés in a single base layer, and reveal them by rectilinear gratings, we can create the three single-moiré bases with gratings at angles of $0^{\circ}$ (similar to Figure 1c), $60^{\circ}$ and $120^{\circ}$ and multiply them together. In the example illustrated in Figure 2, we have designed level-line moirés that are revealed by one grating of a specific period at three different rotation angles (one for each moiré). Instead of using rotated instances of the same grating, we could also work with several periods, orientations and geometric transformations of the gratings. Once one of the revealers is superposed on the resulting base, the corresponding moiré appears. However, since the overall base is a superposition of the three single-moiré bases, it becomes darker when increasing the number of embeddings.

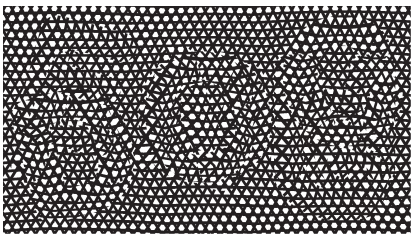

a

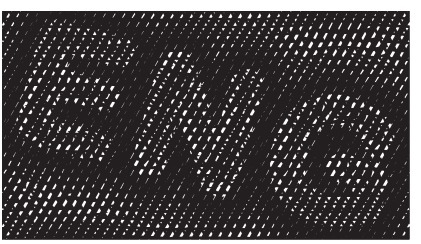

c

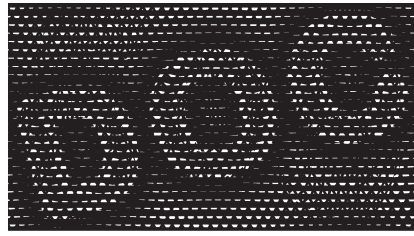

$\mathrm{b}$

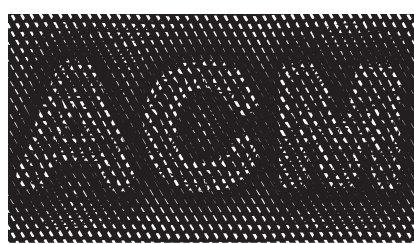

d
Figure 2: (a) Overall base obtained by superposing the three single-moiré bases in a single image. (b), (c) and (d) Levelline moirés obtained when superposing the revealer formed of line gratings at respectively $0^{\circ}, 60^{\circ}$ and $120^{\circ}$.

As an alternative method, one may tile the space and dedicate a portion of the tiles to each moiré layer. Consequently, the intensity of the base remains unchanged and the hidden information is no longer spread on the whole base but only on certain portions of it. Due to the sparsity of the base, the information cannot be decoded with the naked eye.

The method proposed in this paper therefore relies on tessellating the base in a regular manner. Each tile is filled with the corresponding area from one of the single-moiré bases. With this alternative method, a few challenges have to be met. In order to obtain the desired number of well-visible level-line moirés, one needs to select an appropriate tessellation of the space along with well-thought orientations and periods of the revealing layer.

\section{TESSELLATION OF THE SPACE}

Many types of tessellations exist. Since we would like all the hidden moirés to be treated similarly, we limit ourselves to regular tilings. The choice has to be made between the only three regular tilings, which are the triangular, the square and the hexagonal tilings [3, Section 1.3]. In order not to

favour specific line orientations, we should use tiles that are as close as possible to disks. This is why we decided to use a regular hexagonal tiling. Once the hexagonal tiling is created, we need to assign the hexagons to their moiré and therefore obtain the corresponding tessellation. The tessellations for 3, 5, 7 and 9 embeddings can be seen in Figure 3. For 4, 6, 8 and any other number of embeddings, the tessellations can be created in the same manner. A specific hexagon colour is assigned to each single-moiré base. Once the tessellation is created with hexagons of the desired size, each set of coloured hexagons is used as a mask. The masked areas are filled with the corresponding parts of the associated single-moiré base. Even though in some tessellations the layout of the hexagons is not exactly the same for all the embeddings (e.g. in the case of five embeddings), their distribution is nonetheless close to uniform.

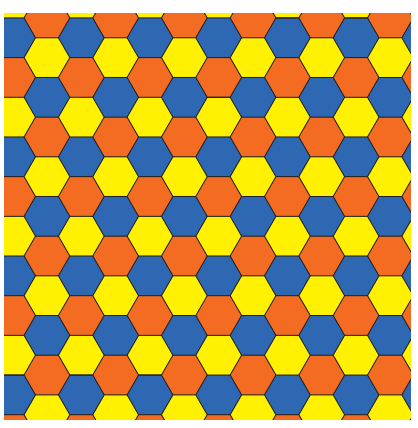

a

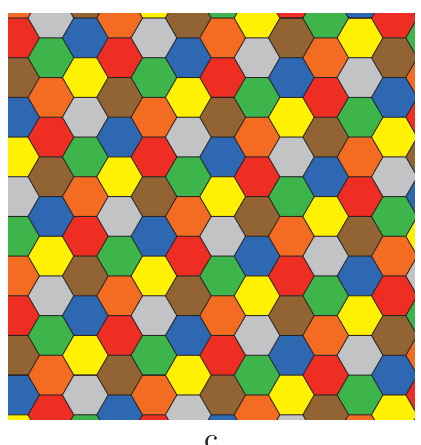

Figure 3: Tessellations for (a) 3, (b) 5, (c) 7 and (d) 9 embeddings.

\section{TRADE-OFFS: GENERAL CONSIDERA- TIONS}

Going from one to $n$ level-line moirés in a single base has an effect on the visibility of the moirés. Only $1 / n$ of the surface can be assigned to each level-line moiré. While one moiré is revealed on a fraction of the space, the other areas do not show any useful information and, to some extent, mask the revealed moiré. Moreover, the sparsity of the revealed tiles also decreases the visibility of the moiré messages since the sampling by the hexagons can be of rather low frequency compared to the typical sizes of the moiré-shape features. In order to embed as many level-line moirés as possible and to maximise their visibility, trade-offs must be found with respect to the size of the hexagons, the revealing period and the size of the smallest moiré-shape feature. 


\section{DIMENSIONS OF THE HEXAGONS AND OF THE LINE GRATINGS}

The revealing period is directly constrained by the fabrication or the printing technology. Concerning the tiles, we would like to have them small enough to provide a high enough sampling of each moiré (see Figure 4). In order to render the moiré properly, the distance between two tiles sampling the same elevation profile must be shorter than a quarter of the size of the moiré's smallest feature (see Figure 5).

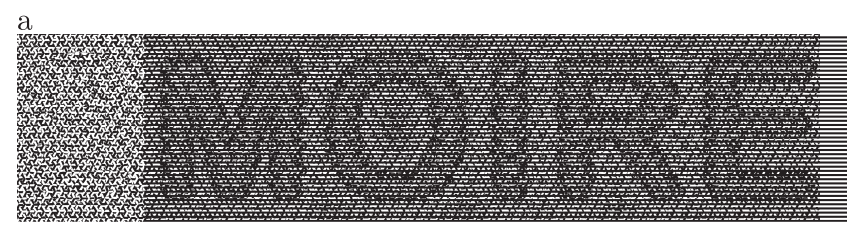

$\mathrm{b}$
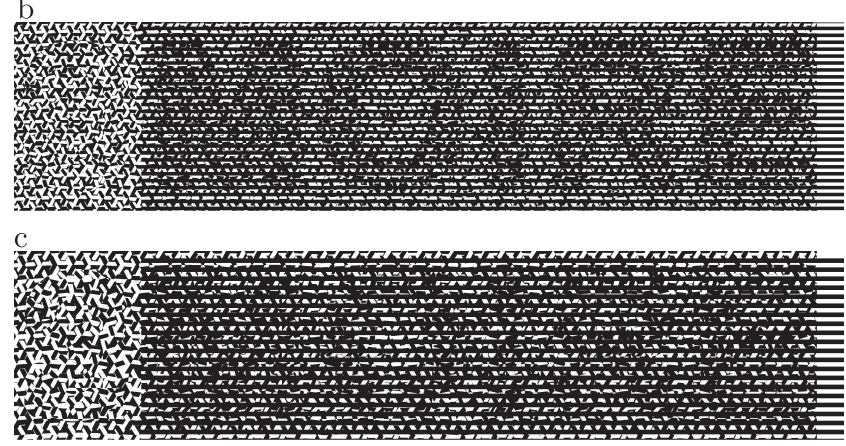

Figure 4: Level-line moirés embedding 3 hidden messages created with hexagons of side-length equal to (a) 12 , (b) 20 and (c) 26 pixels and with a line-grating period of (a) 20, (b) 33 and (c) 43 pixels at 1200dpi. In these images, the left part represents the base alone, the central part represents the superposition of the base and the revealer and the right part represents the revealer alone. The visibility of the moiré decreases as the sampling becomes coarser.

If the tiles are farther from each other, it may still be possible to visually decipher the message. However, parts of the information present in the elevation profile may be lost. Since the distance between two tiles encoding the same elevation profile depends on the number of embeddings (see Figure 3) and on the size of the tiles, these two parameters must be selected in a combined manner. As we increase the number of embeddings, the distance between the hexagons sampling one of the moirés also increases. If we keep the same hexagonal tiles, the limit of a quarter of the size of the moiré's smallest feature can be exceeded. For example, in Figure 6c, it is not possible to correctly embed the 7 different level-line moirés with the chosen tile size. The hexagons are too far apart and we cannot perceive the moiré.

To embed more elevation profiles, the tiles must be of smaller size. In addition, each hexagonal tile needs to cover the line shift that is proportional to the local value of the elevation profile. For a given revealing period, reducing the size of the hexagons decreases the number of periods that can be seen in a tile. Hexagons must contain at least one revealing period and should be as small as possible. As a general rule, the length of the sides of the hexagons should be at
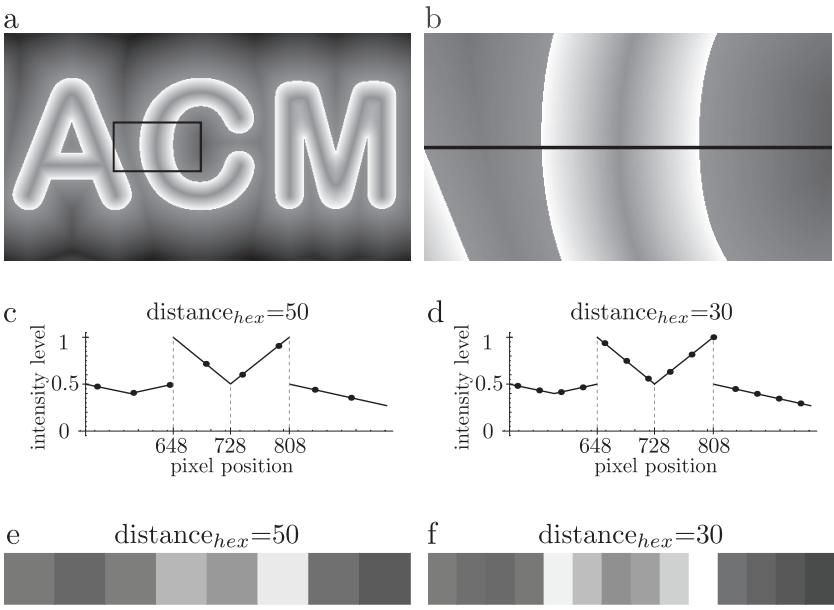

Figure 5: (a) One of the elevation profiles used to create the moiré of Figure 2. (b) Enlargement of the part of (a) with the horizontal line representing the pixels shown in the plots of (c) and (d). (c), (d) Plot representing the elevation profile of the cross-section shown as a line in (b) and the positions where the hexagons sample it, represented by dots, for a distance between hexagons sampling the same moiré equal to 50 pixels, respectively 30 pixels. (e), (f) Resulting sampled elevation-profile values.

least equal to half of the revealing period. This way, one period of the base layer grating will always fit in the hexagons, independently of the orientation. Nevertheless, it is not necessary to always consider the smallest tile dimension. If the moiré's features are large enough, we can also use larger tiles and include more than one period per hexagon. This will lead to a slightly different texture. Similarly, in some applications one may use larger revealing periods in order to obtain a slower displacement of the level lines when moving the revealer. Indeed, the whole cycle of displacement of the level lines is achieved upon translation of the revealer by one period.

\section{CONCLUSION}

The method that we propose in order to embed more than two level-line moirés within a single base-layer is both effective and efficient. The hidden messages cannot be visually deciphered from the base alone and there are some ways to make it also really hard or even impossible to decipher them by a computer, for example by using revealers that are more sophisticated than simple rectilinear gratings. Another advantage of this method compared to the regular single level-line moiré is that we can use the multiple-moiré embedded bases that we create as dither matrices for halftoning. We can then produce greyscale images incorporating several hidden messages that have the shape of level-line moirés without giving any information about the presence of hidden messages within the apparently random structure of the base (see Figure 7). The described features are independent of the technology. With the current print-based technology, we can easily hide 7 layers and have clearly visible levelline moirés. In the future, higher printing resolution and accuracy may allow hiding even more layers of information. 


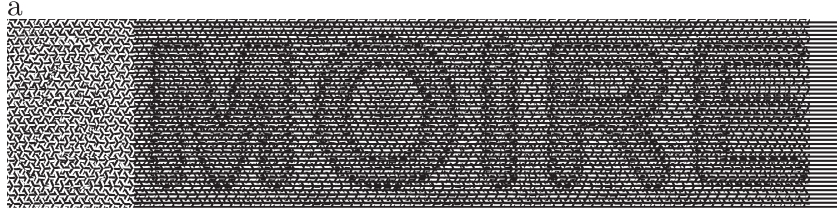

$\mathrm{b}$
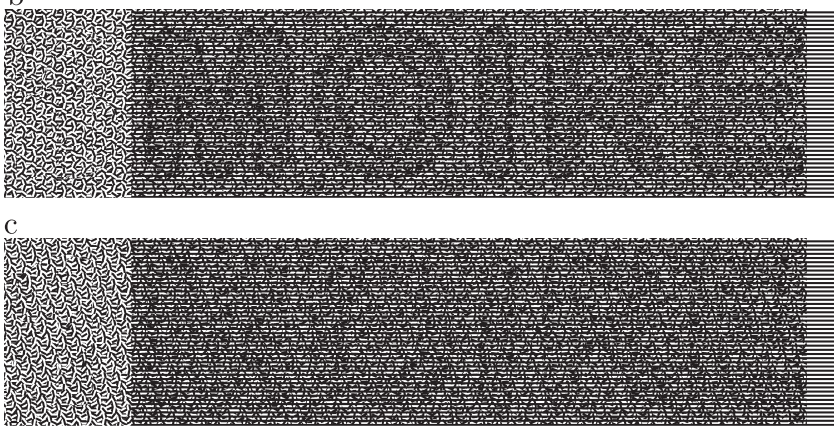

Figure 6: Level-line moirés obtained with hexagons of sidelength equal to 16 pixels and with a line-grating period of 20 pixels at 1200dpi. They embed (a) 3, (b) 5 and (c) 7 hidden messages. In these images, the left part represents the base alone, the central part represents the superposition of the base and the revealer and the right part represents the revealer alone. The feature size to be revealed, e.g. the bar of the M, is of 165 pixels. Therefore, the distance between two tiles sampling the same elevation profile must be smaller than 41 pixels. In these examples, this distance is equal to (a) 16, (b) 28 and (c) 42 pixels. This explains why the moiré is clearly visible in (a) and (b) and only barely visible in (c).

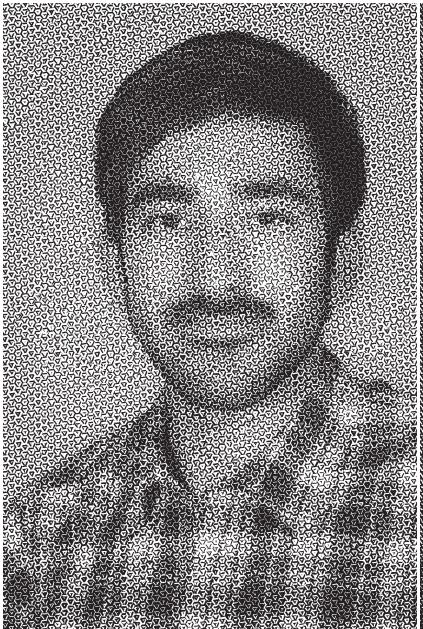

a

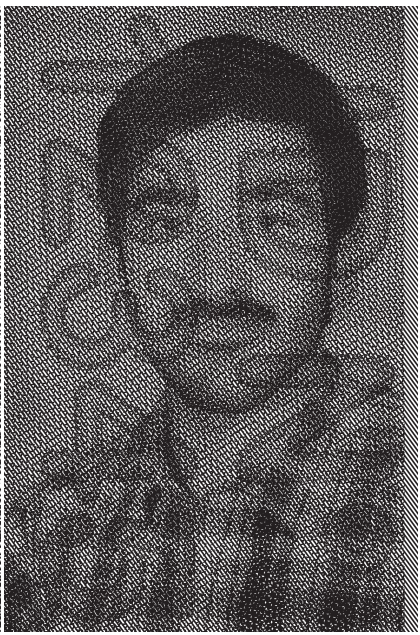

$\mathrm{b}$
Figure 7: (a) Image that has been halftoned to incorporate the desired texture that generates the three level-line moirés when a rectilinear revealer of period 16 pixels at $1200 \mathrm{dpi}$ is superposed on top of it. (b) The level-line moiré revealed with the revealer at $126^{\circ}$. The other hidden moirés can be revealed by orienting the revealer from Figure $8 \mathrm{a}$ at $6^{\circ}$ and $66^{\circ}$.

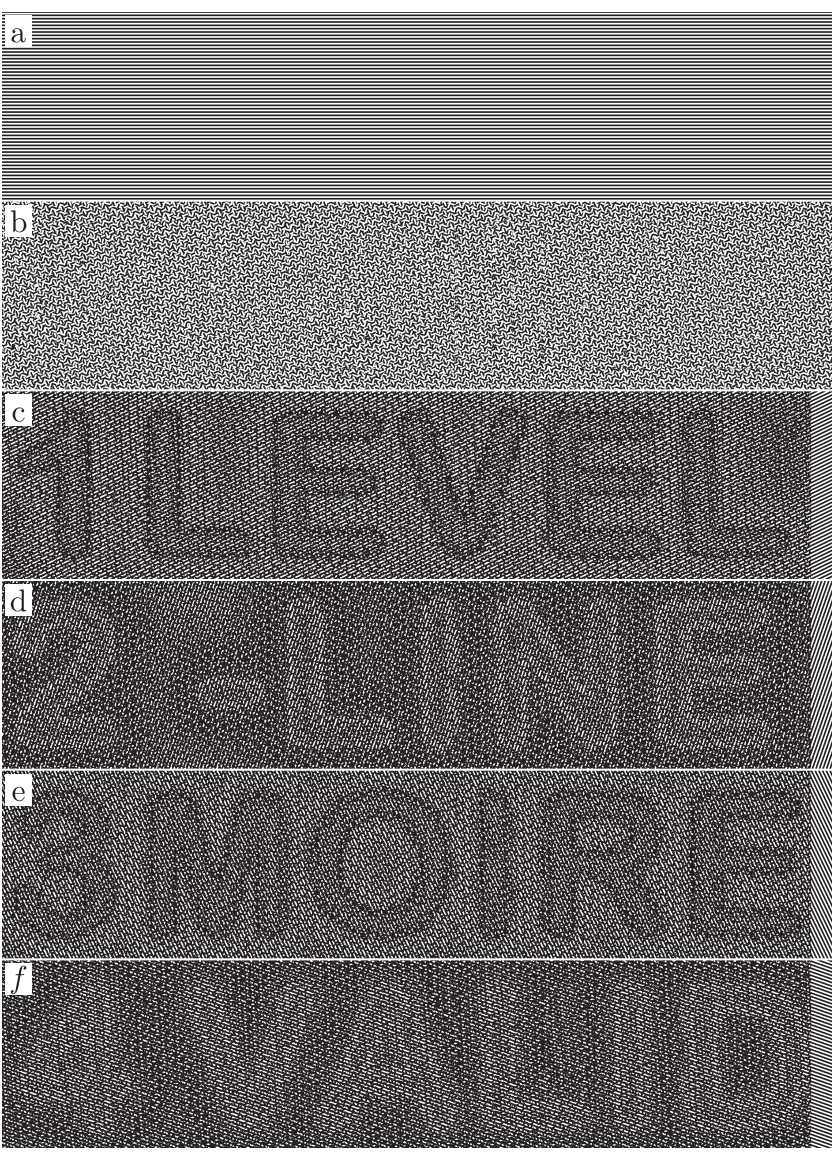

Figure 8: Level-line moirés obtained with hexagons of sidelength equal to 12 pixels and with a line-grating period of 16 pixels at $1200 \mathrm{dpi}$. To visualize the moirés, you can print this page on a paper sheet and on a transparency and superpose the revealer (a) that is printed on the transparency and the base (b) that is printed on the paper sheet. You should then be able to see the moirés if you orient the revealer at (c) $36^{\circ}$, (d) $81^{\circ}$, (e) $126^{\circ}$ and (f) $171^{\circ}$. Once the moiré appears, you can translate the revealer in its direction of periodicity in order to see the dynamic beating effect. The images should be printed at $1200 \mathrm{dpi}$ to obtain the best results.

\section{REFERENCES}

[1] I. Amidror. The theory of the moiré phenomenon. Springer, 2000.

[2] S. M. Chosson and R. D. Hersch. Beating shapes relying on moiré level lines. ACM Trans. Graph., 34(1):9:1-9:11, Dec. 2014.

[3] B. Gruenbaum and G. Shephard. Tilings and patterns: an introduction. New York: Freeman, 1989.

[4] R. D. Hersch and S. M. Chosson. Band Moiré Images. ACM Trans. Graph., 23(3):239-247, Aug. 2004.

[5] G. Lebanon and A. M. Bruckstein. Variational approach to moiré pattern synthesis. J. Opt. Soc. Am. A, 18(6):1371-1382, Jun 2001.

[6] G. Oster. Optical art. Appl. Opt., 4(11):1359-1369, Nov. 1965.

[7] P.-H. Tsai and Y.-Y. Chuang. Target-driven moire pattern synthesis by phase modulation. ICCV '13, pages 1912-1919, 2013. 